\title{
The high pressure gas scintillation proportional counter on-board the BeppoSAX X-ray astronomy satellite
}

\author{
G. Manzo, S. Giarrusso, A. Santangelo, F. Ciralli, G. Fazio, S. Piraino, and A. Segreto \\ CNR - Istituto di Fisica Cosmica ed Applicazioni dell'Informatica, Via Mariano Stabile 172, 90139 Palermo, Italy
}

Received May 15; accepted December 2, 1996

\begin{abstract}
In this paper we describe the High Pressure Gas Scintillation Proportion Counter, one of the Narrow Field Instrument of the X-ray astronomy satellite BeppoSAX. Sensitive in the $4-120 \mathrm{keV}$ band, the HPGSPC will study all astrophysical sources emitting in the poorly studied hard X-ray band. The design and performances of the instrument are reported together with results of the on-ground calibration data analysis.
\end{abstract}

Key words: instrumentation: detectors $-\mathrm{X}$-ray: general

\section{Introduction}

The Italian Dutch Satellite for X-ray Astronomy SAX, Satellite Astronomia X (Scarsi 1993), renamed BeppoSAX in honour of Giuseppe Occhialini, has been carried into a low-Earth orbit (height of $600 \mathrm{~km}$ and inclination $\leq 4^{\circ}$ ) by an Atlas Centaurus Rocket on April 30, 1996. The BeppoSAX payload consists of four Narrow Field Instruments (NFIs) coaligned along the $Z$ axis of the spacecraft and two Wide Field Cameras (WFCs, Jager et al. 1995) which point along the $+y$ and $-y$ axis respectively. The NFIs package includes an imaging Low Energy Concentrator System (LECS, Martin et al. 1995), three units of the imaging Medium Energy Concentrator System (MECS, Boella et al. 1995), the High Pressure Gas Scintillation Proportional Counter (HPGSPC, Giarrusso et al. 1995) and the Phoswhich Detector System (PDS, Frontera et al. 1995). Using the NFIs BeppoSAX will be able to perform detailed spectroscopic and timing studies of more than 2000 celestial X-ray sources with an unprecedented wide energy band that ranges from a fraction of $\mathrm{keV}(0.1 \mathrm{keV}$ is the lower limit of the LECS) up to $300 \mathrm{keV}$ (the upper limit of the PDS). More details on the

Send offprint requests to: A. Santangelo:

andrea@ifcai.pa.cnr.it mission and its scientific objectives can be found in Scarsi (1993) and Piro et al. (1995).

Observation of cyclotron features, that are present in the X-ray spectrum of many celestial sources is the primary scientific goal of the HPGSPC. Cyclotron features provide a powerful means of understanding the physics of radiative transport in highly magnetic plasmas. We note that Ginga observations (Makishima et al. 1991) of cyclotron lines in several accreting X-ray pulsars have confirmed and enlarged previous positive detections by other experiments in Her X - 1 (Trümper et al. 1978) and X0115 + 634 (Wheaton et al. 1979), and have provided a great impetus for attempts at modelling the emission region of these objects. With the HPGSPC and PDS, BeppoSAX will be able to address open crucial points such as the presence and nature of multiple harmonics and the shape and energy of lines. Sensitive in the $4-120 \mathrm{keV}$ energy band the HPGSPC will, also, complement the source continuum observation of concentrators/spectrometers and the PDS and the iron line studies of concentrators/spectrometers. In the anticipated 4 year lifetime of the mission the HPGSPC will study the hard band of the spectrum of many astrophysical objects such as the Seyfert 2 galaxies, some of which have been already detected by OSSE and/or BATSE in the hard X-ray band (Johnson et al. 1994), and Seyfert 1 galaxies (to study the high energy bump above $10 \mathrm{keV}$ and the presence of the break above about $50 \mathrm{keV}$ ). In addition the HPGSPC will address the problem of the hard tails in the spectrum of black hole candidates.

Funded by the Italian Space Agency (ASI), the HPGSPC was invented and designed by the Istituto di Fisica Cosmica ed Applicazioni dell'Informatica (IFCAI, Palermo) of the Consiglio Nazionale delle Ricerche and has been developed by Laben, Milan and Alenia Spazio, Turin, under the scientific responsibility of IFCAI and ASI.

Both the Flight Model and the Spare Flight Unit have been successfully integrated, tested and calibrated at the Laben premises respectively during October/November 1994 and July/August 1995. 


\section{Instrument description}

The HPGSPC instrument is a High Pressure Gas Scintillation Proportional Counter filled with a high purity gas mixture of Xenon (90\%) and Helium (10\%) at 5 atmospheres.

The basic mechanism of Gas Scintillation Proportional Counters is quite well known and has been extensively described by different authors (Gedanken et al. 1972; Manzo et al. 1980).

After penetrating the beryllium window, the X-ray photon is absorbed in the gas cell by a Xenon atom via the photoelectric effect. The primary photoelectron emitted gives rise to a localised cloud of secondary electrons at the position of X-ray absorption. The secondary electron cloud, driven by a relatively moderate electric field, after drifting in the so called Drift Region, enters a high field region (Scintillation Region) in which the electrons acquire sufficient energy to excite the Xenon atoms. As a result of collisions, the excited atoms form excited diatomic Xenon molecules which then deexcite by the emission of VUV photons in the $1500-1950$ angstrom range. Typical duration of scintillation, measured as the time interval between the $10 \%$ and $90 \%$ of the signal, is $3.5 \mu \mathrm{sec}$ and does not depend on the energy. In the case of the HPGSPC VUV light is collected by an array of seven photomultipliers in an Anger Camera configuration. The number of electrons emitted per keV by the cathode of central photomultiplier is about 250 .

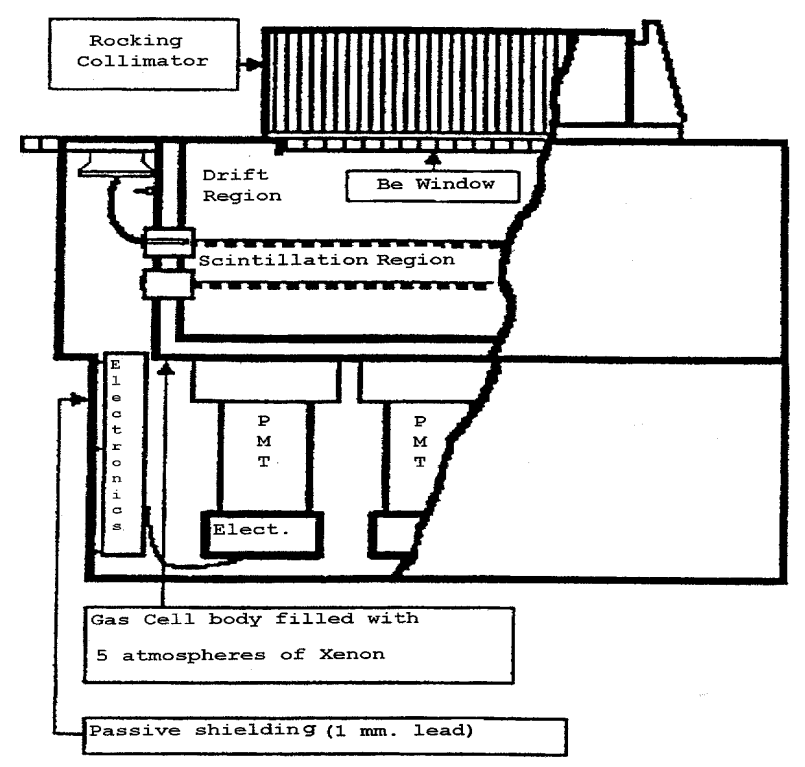

Fig. 1. Schematic view of the Flight model of the HPGSPC

A schematic view of the main components of the Flight Model of the HPGSPC is shown in Fig. 1. Table 1 sum- marises the main characteristics. The HPGSPC instrument consists, primarily, of a Detector Unit (DU) and an Electronic Unit (EU).

\subsection{Detector unit}

The construction of the sealed cylindrical gas cell, whose cross section is shown in Fig. 2, was carried out by AEG, Ulm, following Ultra High Vacuum standards. This is imposed by the requirements on leak tightness $\left(10^{-9} \mathrm{~cm}^{3}\right.$ $\mathrm{He} / \mathrm{s}$ ) and cleanliness of the filling gas (at a few part per million level) for an operational life lasting more than four years. It consists, primarily, of a titanium body $(3 \mathrm{~mm}$ thick, with a diameter of $360 \mathrm{~mm}$ and a depth of 184.5 $\mathrm{mm})$ covered with an entrance window $(30 \mathrm{~cm}$ diameter) made of beryllium. The beryllium window, shown in Fig. 3, was one of the most technically challenging parts of the instrument. The design must meet two contradictory requirements: transparency to $\mathrm{X}$-rays down to $3 \mathrm{keV}$ and the mechanical stiffness required to withstand the 5 atmospheres differential pressure on a diameter of $30 \mathrm{~cm}$. The window is made of two beryllium foils, 0.5 and 0.8 $\mathrm{mm}$ thick supported by an egg crate structure of orthogonal beryllium ribs. The core self supporting structure is welded to the titanium body via an aluminium/titanium transition ring aimed at compensating for mechanical stresses generated during the bake-out at $300^{\circ}$ degrees.

Table 1. HPGSPC overall characteristics

\begin{tabular}{ll}
\hline Parameter & value \\
\hline Exposed geometrical area & $450 \mathrm{~cm}^{2}$ \\
Body of cell & titanium $3 \mathrm{~mm}$ thick \\
Depth of Drift Region & $10 \mathrm{~cm}$ \\
Depth of Scintillation Region & $1 \mathrm{~cm}$ \\
Depth of Back Region & $6.3 \mathrm{~cm}$ \\
Suprasil UV Exit Window & $8.4 \mathrm{~cm}$ diam, $5 \mathrm{~mm}$ thick \\
Suprasil UV read-out & $8.1 \mathrm{~cm}$ diam \\
Drift Region Voltage & $8 \mathrm{kVolt}$ \\
Scintillation Region Voltage & $20 \mathrm{kVolt}$ \\
Filling Gas & $90 \%$ Xe $+10 \% \mathrm{He}$ \\
Filling Pressure & $5 \mathrm{~atm}$ \\
Outer Diameter & $42.5 \mathrm{~cm}$ \\
Useful Diameter & $24 \mathrm{~cm}$ \\
\hline
\end{tabular}

An absorption region (Drift Region) of $10 \mathrm{~cm}$ and a scintillation gap (Scintillation Region) of $1 \mathrm{~cm}$ are defined by two circular mesh grids with a diameter of $300 \mathrm{~mm}$, made with orthogonal titanium wires with a spacing of $3 \mathrm{~mm}$ and a diameter of $150 \mu \mathrm{m}$. To maintain the uniformity of the electric field in the Drift Region five field shaping rings made of NCR alloy are placed every $16.0 \mathrm{~mm}$. The two mesh grids can be set up to a 


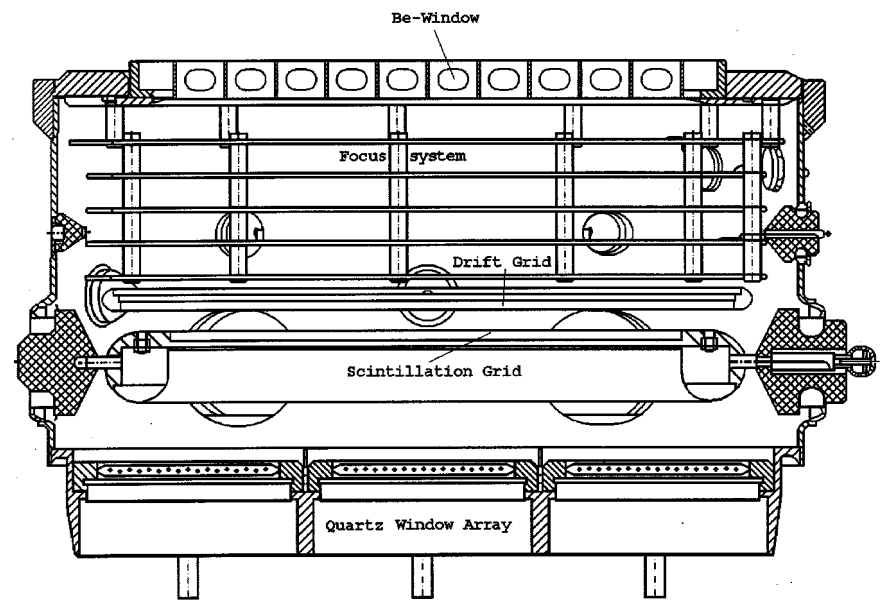

Fig. 2. Gas cell cross section

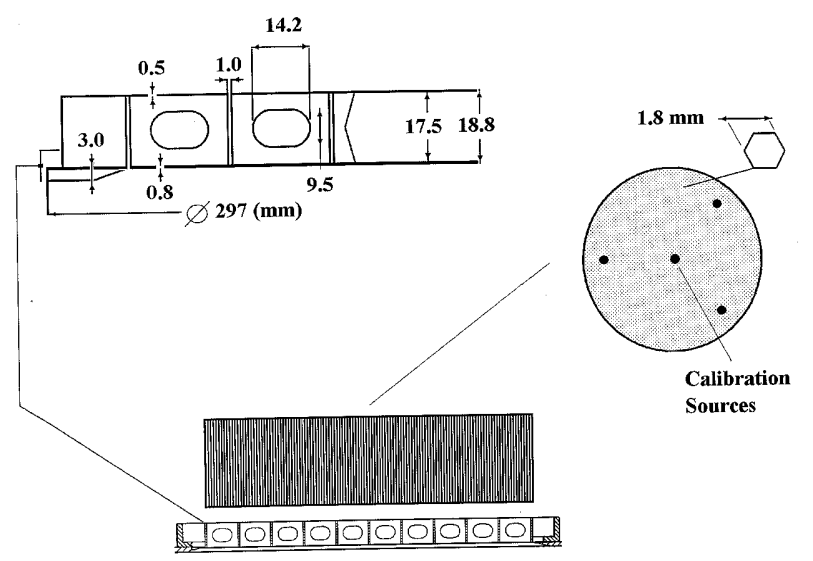

Fig. 3. Collimator and window

nominal value of 10 and $25 \mathrm{kVolt}$. In the Flight Model, however, because of microdischarge occurring in a localised point in one of the high voltage feedthroughs, the reduced field applied in the Scintillation Region was only $2 \mathrm{kVolt} / \mathrm{cm} / \mathrm{atm}$ with a reduction of VUV light production. Due to the high electrical field, the two grids are terminated by guard electrodes whose shape is critical in terms of higher field values generated. Each of the two grids is held in place by six ceramic insulators which are welded to the outer wall of the cell. For each grid one of the insulators is also used as a high voltage feedthrough. The high voltage applied, the constraints on the dimension and shape, the breakdown field strength of the gas have made the design of the grids rather critical. In the end, a compromise between the best theoretical (Rogovsky profile) and the most feasible shape has been used. This is a circular profile with a variable radius.

At the bottom of the detector seven ruggidized EMI D319Q photomultipliers (PMTs) in an Anger camera configuration, as in Fig. 4, detect the VUV light produced

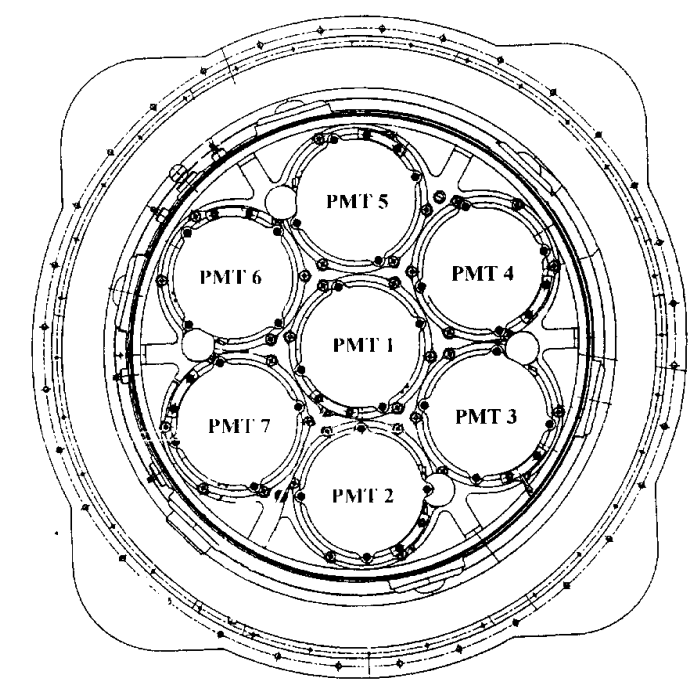

Fig. 4. The PMTs in an Anger camera configuration: the central PMT is surrounded by six lateral PMTs

in the Scintillation Region. As an interface between the PMTs and the pressurized gas cell a titanium flange, welded to the titanium body, supports seven suprasil 1 quartz windows each $5 \mathrm{~mm}$ thick. The $6.3 \mathrm{~cm}$ between the scintillation grid and the quartz windows define the so called "Back Region". On top of the detector a $10 \mathrm{~cm}$ high collimator (manufactured by Officine Galileo, Florence) limits the field of view to $1^{\circ} \times 1^{\circ}$ degree FWHM. The collimator consists of hexagonal cells made with $50 \mu \mathrm{m}$ of aluminium plated with $10+10 \mu \mathrm{m}$ of lead. Four highly collimated calibration sources are mounted in the collimator in the position shown in Fig. 3. Each calibration source consists of a mixture of $\mathrm{Fe}^{55}$ and $\mathrm{Cd}^{109}$ radioactive sources, with calibration lines at $6,22,25$ and $88 \mathrm{keV}$. The sources are used for a continuous monitoring of the gain and for a real time equalization of the relative gain of the photomultipliers performed by the Automatic Gain Control (AGC) chain. To reduce the residual background, the Detector Unit is shielded with $1 \mathrm{~mm}$ of lead and $2 \mathrm{~mm}$ of tin around the sides and bottom.

\subsection{The electronics}

The HPGSPC High Voltage supplies, the Front End Electronic (FEE), the Electronic Unit and the data conditioning system have been designed and produced by Laben, Milan.

The seven signals coming out from the PMTs are slightly preamplified, shaped and then transmitted, via a Current Loop transmitter, from the FEE to the Electronic Unit that constitutes the main electronic part of the 
Instrument. The Electronic Unit interfaces the Detector Unit to the On Board Data Handling (OBDH) bus. In the EU, the PMT signals received from the DU are processed, A/D converted and finally formatted for transmission to the OBDH. In addition, Housekeeping signals (High Voltage monitoring and temperature monitoring) are converted and formatted, jointly with other Digital Housekeeping, for transmission to the OBDH.

The signals coming out from the DU are slightly delayed (this is needed to allow the event qualification board to send the "integrate" signal if the current pulse exceeds a programmable threshold) and then integrated by seven gated integrators each with $8 \mu \mathrm{sec}$ integration time constant. A Sum signal is then obtained with an analogue sum of the seven integrated signals. The pulse duration is also measured by the burst length chain that measures the interval time between the $20 \%$ and the $80 \%$ of the integrated sum signal. As shown below, information on burst length is used to discriminate genuine $\mathrm{X}$-rays against background events or anomalous events such as those absorbed in the Scintillation Region and/or Back Region.

Event qualification and processing is performed both by the Analogue Processor and the Digital Processor. While analysis of energy and of pulses shape along with burst length qualification and $\mathrm{A} / \mathrm{D}$ conversion is performed by the Analogue Processor, two main tasks are performed by Digital Processor: single and double event management and the position reconstruction and energy correction of the events. These tasks, as discussed in the next paragraphs, are crucial for the correct scientific working of the Instrument.

A real time equalisation of the 7 PMTs is performed on-board by the Automatic Gain Control System. For each event that is qualified as a $22 \mathrm{keV}$ photon from the central $\mathrm{Cd}^{109}$ calibration source the channel value measured by each Photomultiplier is compared with the correspondent reference channel experimentally determined during on-ground calibration. If the measured channel is lower than the reference channel the gain of the PMT is increased by a step $\Delta G$ acting on the voltage of the phototube. On the contrary if the measured channel is greater than the reference channel the gain of the phototube is changed decreasing by a single step the voltage applied to the phototube. For each PMT a gain variation of $0.1 \%$, which corresponds to $100 \mathrm{mVolt}$, has been chosen to maintain overall fluctuations in $\mathrm{Cd}^{109}$ peak position reconstruction around 0.5\% FWHM. This choice, given the actual calibration source count rate, allows equalisation of the system in less than a minute without degrading the energy resolution.

\subsection{The HPGSPC data structure}

The HPGSPC instrument can be operated in three different telemetry modes:
- Direct Mode in which information is transmitted for every detected and qualified photon. Five Direct Mode transmission option are foreseen: Table 2 reports a list of the parameters transmitted with the relative telemetry for each Direct Mode.

- Indirect Mode in which energy and burst length spectra for single and correlated events are generated. For these integrated spectra the number of energy channel (1024 for energy, 256 for burst length spectra), the maximum content of each channel ( 8 or 16 bits) and the accumulation time can be independently set.

- Indirect Temporal mode in which, beside the spectra generated in Indirect Mode, energy spectra accumulated in 1 to 4 energy bands on time scales down to $1 \mathrm{msec}$, are produced.

The total amount of telemetry allocated for the HPGSPC is $50 \mathrm{kBit} / \mathrm{sec}$ and can go up to $100 \mathrm{kBit} / \mathrm{sec}$ if all the other instruments are in a standby position. Due to telemetry constraints and depending on the source count rate not all the information generated by the EU for every event can be transmitted. The main observing mode is, therefore, the Dir001 mode while the Dir004 is considered the diagnostic operative mode. The latter has been extensively used during the on-ground calibration and during the Commissioning and Science Verification Phase to configure the instrument optimally and to determine its response characteristic in flight. It will, also, be used during periodic calibration of the instrument or to verify the correct performances of the PMTs.

Whatever Operative Mode is selected, other information is telemetered to the ground to monitor the detector status and performance:

- Calibration Spectra, four spectra (one for each Calibration Source), generated every 128 seconds.

- Ratemeters to check the AC logic and Dead Time.

- Engineering HouseKeeping files which contain: ON/OFF status, Digital Thresholds, Temperatures, Voltages, Collimator Status and Position.

\section{Principle of operation}

\subsection{Fluorescence gated technique}

Photoabsorption of $\mathrm{X}$-rays below the Xenon $K$-binding energy at $34.5 \mathrm{keV}$, occurs in the $L$ or lower order shells. In this case only a single cloud is produced for each $\mathrm{X}$-ray event, because the probability of atomic relaxation via the Auger effect is much higher than via fluorescence emission and in any case the mean penetration depth of the $L$ fluorescence photon, in 5 atmospheres Xenon, is less than $1 \mathrm{~mm}$. For each X-ray event with $E_{\mathrm{x}} \leq 34.5 \mathrm{keV}$ only a single VUV burst will be produced and then detected by the HPGSPC.

For incident $\mathrm{X}$-ray energies above $34.5 \mathrm{keV}$, the probability that photoabsorption occurs in the $\mathrm{K}$ shell is 
Table 2. HPGSPC telemetry of direct modes

\begin{tabular}{llllll}
\hline Parameter & DIR001 & DIR002 & DIR003 & DIR004 & DIR005 \\
\hline Arrival Time & $16 \mathrm{bit}$ & $16 \mathrm{bit}$ & $16 \mathrm{bit}$ & $16 \mathrm{bit}$ & $16 \mathrm{bit}$ \\
Uncorrected Energy & & & $12 \mathrm{bit}$ & $12 \mathrm{bit}$ & $12 \mathrm{bit}$ \\
Corrected Energy & $12 \mathrm{bit}$ & $12 \mathrm{bit}$ & $12 \mathrm{bit}$ & $12 \mathrm{bit}$ & $12 \mathrm{bit}$ \\
Burst length & $8 \mathrm{bit}$ & & $8 \mathrm{bit}$ & $8 \mathrm{bit}$ & $8 \mathrm{bit}$ \\
7 PMTs signals & & & & $7 * 12 \mathrm{bit}$ & $3 * 12$ \\
Position in sector & & & $12 \mathrm{bit}$ & $12 \mathrm{bit}$ & $12 \mathrm{bit}$ \\
Sector identity & $1 \mathrm{bit}$ & $1 \mathrm{bit}$ & $1 \mathrm{bit}$ & $1 \mathrm{bit}$ & $1 \mathrm{bit}$ \\
Correlation Flag & $1 \mathrm{bit}$ & $1 \mathrm{bit}$ & $1 \mathrm{bit}$ & $1 \mathrm{bit}$ & $1 \mathrm{bit}$ \\
Calibration Flag & $2 \mathrm{bit}$ & $2 \mathrm{bit}$ & $2 \mathrm{bit}$ & $2 \mathrm{bit}$ & $2 \mathrm{bit}$ \\
Calibration identity & $40 \mathrm{bit}$ & $32 \mathrm{bit}$ & $68 \mathrm{bit}$ & $152 \mathrm{bit}$ & $100 \mathrm{bit}$ \\
Total telemetry & & & &
\end{tabular}

$86 \%$ while the probability that atom relaxes via a $K_{\alpha}$ $(29.7 \mathrm{keV})$ or a $K_{\beta}(33.8 \mathrm{keV})$ fluorescence photon is as high as $87 \%$. Depending on geometry, filling pressure and energy of the original $\mathrm{X}$-ray event the fluorescence photon can be reabsorbed in the gas cell, generating a second localized electron cloud at a different point respect to the primary photoelectron cloud. The two electron clouds from the double interaction (from now on referred as residual and fluorescence) will enter the Scintillation Region with a time difference of $40 \mu \mathrm{s}$, limited by the maximum drift time in the Drift Region. In this case, a double VUV light burst associated to a single X-ray event will be produced and detected by the HPGSPC. The detection of a $K_{\alpha}$ or $K_{\beta}$ photon will "gate" the partial photoabsorption.

$\mathrm{X}$-ray events above $34.5 \mathrm{keV}$ can, however, interact with the $L$ shell or interact with the $K$ shell and the atom relaxes via an Auger electron. In these cases only a single cloud is produced. Finally, for X-ray events that give rise to a $K_{\alpha}$ or $K_{\beta}$ fluorescence photon a single event can still be detected if the two clouds are not spatially resolved or when the fluorescence photon escapes the detector leaving only the residual energy deposit of $E_{\mathrm{x}}-E_{\mathrm{b}}$, where $E_{\mathrm{b}}$ is the binding energy and $E_{\mathrm{x}}$ is the energy of the incident $\mathrm{X}$-ray.

It has been suggested by many authors that the detection of the double events may provide a unique signature for a true X-ray event and, in this sense, can be used to discriminate against a non X-ray background event (Manzo et al. 1980; Ramsey et al. 1990; Dangendorf et al. 1989).

The "Fluorescence Gated" technique can also be used to improve the energy resolution above the Xenon $K$ shell (Taylor et al. 1981). Since the energy of the fluorescence photon is exactly known, only the statistical fluctuations relative to the clouds of primaries (residuals) will affect the energy determination.

\subsection{Scientific modes of operation}

Following the physics of photoabsorption three different scientific modes have been defined for the HPGSPC and implemented during the on ground data analysis:

\section{- Single Event Mode (SE) \\ - Fluorescence Gated Mode (FG) \\ - All Events Mode (All).}

In $\mathrm{SE}$ mode only $\mathrm{X}$-ray photons detected as a single VUV light burst are collected. The SE mode can be used in the HPGSPC whole range and is the only mode for $\mathrm{X}$-ray events with energy below $34.6 \mathrm{keV}$. In FG mode only incident $\mathrm{X}$-rays producing double correlated events are accumulated. The FG mode is used only above $34.6 \mathrm{keV}$. In the "All" mode both "Single" and "Double correlated" events are accumulated. Defined in the entire energy band of the HPGSPC, the "All" mode maximizes the detection efficiency of the Instrument.

\subsection{Position reconstruction and energy correction}

The HPGSPC energy resolution for a well collimated "on axis" X-ray beam is determined in the first approximation by the statistical fluctuations in the number of electrons produced in the absorption region and the statistical fluctuations in the number of VUV photons detected by the PMTs (i.e. the photon counting statistics). In the case of the HPGSPC, considering a Fano factor of 0.15 (Anderson et al. 1979) and the VUV light yield as reported in Nguyen et al. (1980), a theoretical value for the energy resolution of $2.5 \%$ at $60 \mathrm{keV}$ is obtained ( in "All" mode).

When the HPGSPC is illuminated over the full geometrical aperture (full area illumination) the energy resolution $\Delta E_{\mathrm{m}} / E_{\mathrm{m}}$ (where $E_{\mathrm{m}}$ is the measured energy i.e. the sum of seven PMTs signals) degrades and only slightly depends on the energy. This is due to the dependence of the measured energy $E_{\mathrm{m}}$ on the $(x, y)$ position at which the primary electron cloud enters the Scintillation Region. 
Indeed, it is the effective solid angle subtended by the seven PMTs (and then the VUV scintillation light collected) that strongly depends on the $(x, y)$ scintillation position. Solid angle can vary from the center of the detector to the walls by up to about $20 \%$ (Giarrusso et al. 1989). The measured energy $E_{\mathrm{m}}$, however, can be corrected to the "on axis" value and the energy resolution can be restored just by multiplying the measured energy $E_{\mathrm{m}}$ by the $K_{\mathrm{c}}$ ratio of the total effective solid angle "on axis" to the total effective solid angle subtended by the PMTs at the $(x, y)$ point:

$E_{\mathrm{c}}=E_{\mathrm{m}} \frac{\Omega_{\mathrm{T}}(0,0)}{\Omega_{T}(x, y)}=K_{\mathrm{c}} E_{\mathrm{m}}$

with

$\Omega_{\mathrm{T}}(x, y)=\sum_{i=0}^{6} \Omega_{i}(x, y)$.

In the previous equation $\Omega_{\mathrm{T}}(x, y)$ is the total solid angle subtended by the seven PMTs at the $(x, y)$ position and $\Omega_{i}(x, y)$ is the solid angle subtended by the PMT i. Although different methods have been derived to determine a statistically optimum estimation of the scintillation position for an array of PMTs operating in an Anger camera configuration, they require difficult to implement onboard hardware, or a long processing time (Giarrusso et al. 1995). The method implemented for onboard position reconstruction and energy correction processing makes use of the signal of the central PMT and the highest signal of the lateral PMTs, to determine for each event two energy independent coefficients which are defined as:

$C_{0}=\frac{\mathrm{PMT}_{0}}{\sum_{\mathrm{i}=0}^{6} \mathrm{PMT}_{\mathrm{i}}}$

and

$C_{1}=\frac{\mathrm{PMT}_{1}}{\sum_{\mathrm{i}=0}^{6} \mathrm{PMT}_{\mathrm{i}}}$

where $\mathrm{PMT}_{0}$ is the signal detected by the central PMT and $\mathrm{PMT}_{1}$ is the highest signal among the lateral PMTs. Because there is an univoque correspondence between the $(x, y)$ position in a given sector and the $\left(C_{0}, C_{1}\right)$ space, once the $C_{0}$ and $C_{\mathrm{l}}$ coefficients are obtained, the $K_{\mathrm{c}}$ correction factor is extracted from a look-up table that is experimentally determined and implemented onboard as a memory matrix of $K_{\mathrm{c}}$ values. Each value in the table is addressed by the $C_{0}$ and $C_{1}$ coefficients measured for the event. To make the determination of the $K_{\mathrm{c}}$ value faster, the look-up table is built using the isocoefficients $C_{0}$. Thus the look-up table is defined $C_{0}$ constant map. More details on the position reconstruction and energy correction of the event can be found in (Giarrusso et al. 1995; Giarrusso et al. 1989).

\section{Instrument performance}

During the on-ground calibration measurements, performed at Laben in Milan, the DU-EU assembly was connected to the Instrument Test Equipment (ITE) developed by Laben. The ITE is a computer controlled system with a probe, the On Board Data Handling Bus Emulator and Probe, that emulates the satellite OBDH bus. It retrieves data from the HPGSPC-EU in the form of telemetry packets and send those data to a VAX station via TCP/IP protocol. The on-line data handling, the off-line processing and archival of data from ITE were performed by the IFCAI calibration support system. A two axis PC controlled translation stage was used to position collimated radioactive sources in the relevant point of the entrance window of the detector. Test and calibration of the HPGSPC were performed using four different radioactive sources. Sources and relevant lines are given in Table 3. During the collimated measurements graded shield collimators (made of $\mathrm{Pb}-\mathrm{Sn}-\mathrm{Cu}-\mathrm{Al}$ ) were used. $\mathrm{A} \mathrm{Co}^{60}$ (partially collimated) gamma ray source was also used to simulate the gamma ray induced background events.

Table 3. Calibration sources and lines

\begin{tabular}{lll}
\hline Source & line & Energy $(\mathrm{keV})$ \\
\hline $\mathrm{Fe}^{55}$ & $\mathrm{Mn} K_{\alpha}$ & 5.9 \\
$\mathrm{Cd}^{109}$ & $\mathrm{Ag} K_{\alpha}$ & 22 \\
$\mathrm{Cd}^{109}$ & $\mathrm{Ag} K_{\beta}$ & 25 \\
$\mathrm{Am}^{241}$ & Nucl. line & 59.5 \\
$\mathrm{Co}^{57}$ & Nucl. line & $14,122,136$ \\
$\mathrm{Co}^{60}$ & gamma & 1170,1330 \\
\hline
\end{tabular}

\subsection{Burst length analysis}

Burst length discrimination is a well established method to reduce the background counting rate in GSPCs. The method, that in the case of the HPGSPC is based on the ARC/CFT electronic chain (Leimann \& Van Dordrecht 1979), essentially discriminates the pulse time duration produced by the localised electron cloud generated by the absorption of a genuine X-ray, against the extended ionisation track induced by background particles interacting in the gas. Also background events generated close to the detector walls by the interaction of gamma rays or particles in the detector shielding are discriminated because of their very short burst lengths.

For each event, the $20-80 \%$ rise-time of the integrated sum signal is measured. Only events within the experimentally determined upper and lower thresholds are recognised as potential genuine $\mathrm{X}$-ray events. The full area burst length spectra relative to $\mathrm{Cd}^{109}$ and $\mathrm{Am}^{241} \mathrm{X}$-ray sources 
are shown in Figs. 5 and 6 . The $\mathrm{Cd}^{109}$ spectrum follows a Gaussian distribution with a peak value at $3.74 \mu$ s and a resolution of $8.3 \%$ FWHM. The burst length spectrum of $59.5 \mathrm{keV}$ photons of $\mathrm{Am}^{241}$ is asymmetric with an extended tail towards longer burst lengths. The peak position is at $3.73 \mu$ s and resolution is $8.6 \%$ FWHM. The tail is due to events in which the residual and fluorescence clouds overlap, scintillating for a longer duration.

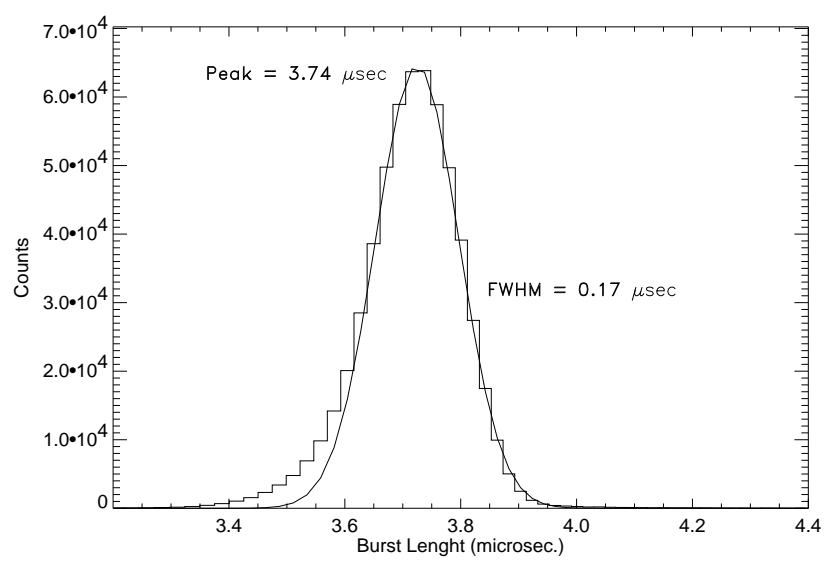

Fig. 5. Burst length spectrum of the 22 and $25 \mathrm{keV}$ lines of $\mathrm{Cd}^{109}$ radioactive source. Continous line is the best gaussian fit to the experimental measurement

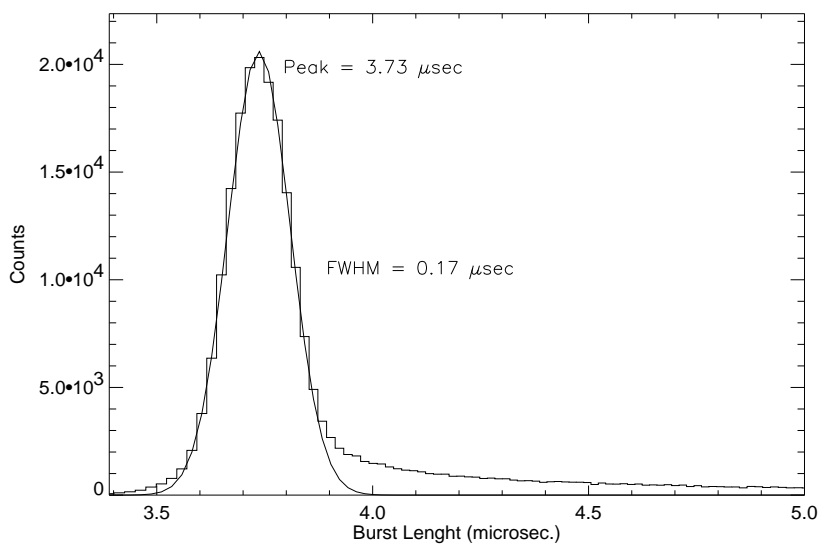

Fig. 6. Burst length spectrum of the $59.5 \mathrm{keV}$ line of $\mathrm{Am}^{241}$ radioactive source. The long tail towards longer burst lengths is due to events which give rise to overlapping residual and fluorescence electron clouds

In Fig. 7 we show the burst length versus energy diagram for the 22 and $25 \mathrm{keV}$ line of $\mathrm{Cd}^{109}$. Events labeled with (1) are associated with Cadmium events absorbed in

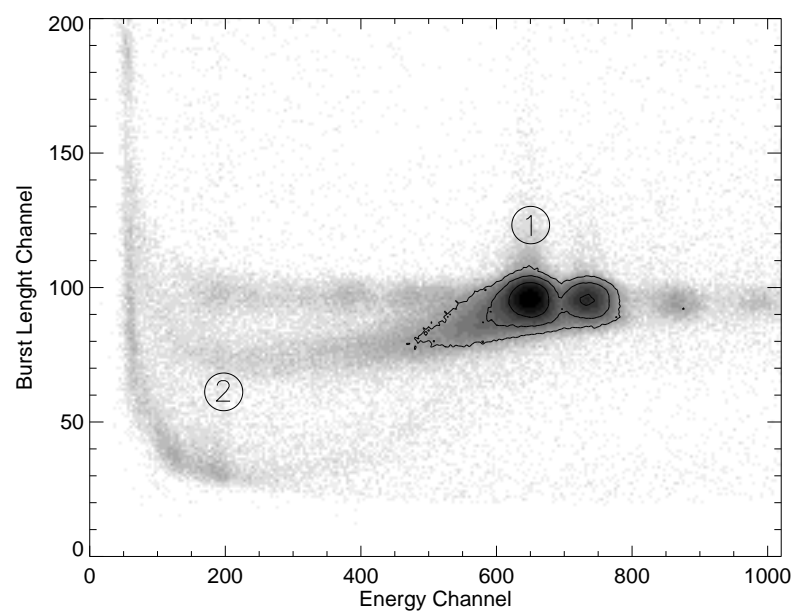

Fig. 7. Burst length versus energy diagram for 22 and $25 \mathrm{keV}$ photons from $\mathrm{Cd}^{109}$ radioactive source. See the text for a description of the labels. Scale is logarithmic

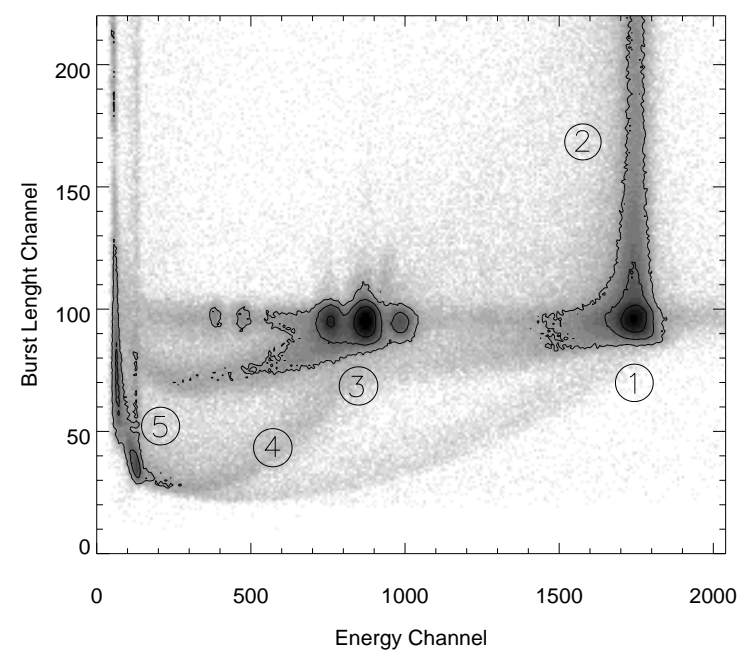

Fig. 8. Burst length versus energy diagram for $59.5 \mathrm{keV}$ photons from $\mathrm{Am}^{241}$ radioactive source

the Drift Region while the extended tail (2) is correlated with events that penetrated deep into the Scintillation Region. These events, scintillating for a shorter time, give rise to a detected events with lower energy and burst length. For incident photons with energies above the Xenon $K$-shell the situation is more complex. Figure 8 is an exposure at $59.5 \mathrm{keV}$ photons from $\mathrm{Am}^{241}$ radioactive source. The events labeled with (1) are associated with $59.5 \mathrm{keV}$ events that have been detected as "Single" i.e. events originating a single electron cloud. The burst length distribution relative to these events shows a very long tail (2) towards longer burst lengths. As mentioned above, these are events that generated a fluorescence 
photon whose cloud overlaps the residual cloud. Events labeled with (3) are associated with the $K_{\alpha}$ and $K_{\beta}$ fluorescence photons and the $K_{\alpha}$ and $K_{\beta}$ escape peaks. Tails are not present for these events associated with a single electron cloud. Events absorbed in the Scintillation Region give rise to the "strips" (4) that connect the source region with the dense region at lower energy and lower burst length (5). The latter is associated with the low- energy features that are due to $59.5 \mathrm{keV} \mathrm{Am}{ }^{241}$ events absorbed in the Back Region which, scintillating close to the wires of scintillation grid along paths of anomalous electric field, generate small short pulses.

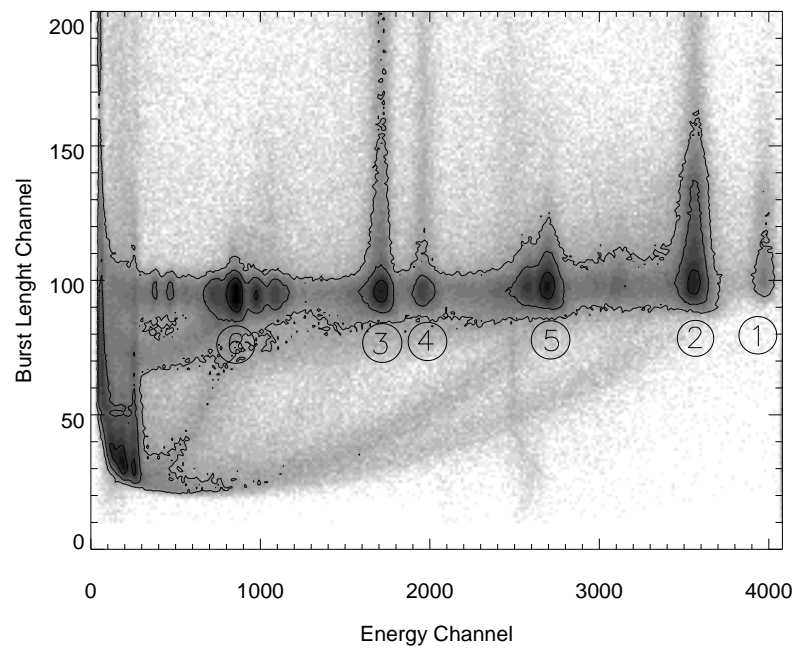

Fig. 9. Burst length versus energy diagram relative to $\mathrm{Co}^{57}$ illumination. Labels are explained in the text

All the features described above are well summarised by the $\mathrm{Co}^{57}$ burst length versus energy diagram shown in Fig. 9. All the "genuine" X-rays peaks are distributed in a very narrow region that run almost parallel to the corrected energy axis, confirming that the burst length distribution is independent of the energy of incident $\mathrm{X}$-rays. Long tails are associated with the primary release plus overlapping fluorescence for the primary lines at 136, 122, 59 and $67 \mathrm{keV}$ of Cobalt (1), (2), (3) and (4) while tails disappear in the peaks at $92.3 \mathrm{keV}$ and $88.3 \mathrm{keV}$ (associated with residuals (5)) and at 29.7 and $33.8 \mathrm{keV}$ (associated with fluorescence photons (6)). The 58.9 and $67.6 \mathrm{keV}$ lines are respectively the $K_{\alpha}$ and $K_{\beta}$ fluorescence photons of the Tungsten (that shielded the Cobalt radioactive source) induced by the 136 and $122 \mathrm{keV}$ photons of the $\mathrm{Co}^{57}$.

In order to reject events absorbed in the Scintillation and Back Region, the burst length lower threshold was set at $3.5 \mu \mathrm{s}$, independent of the energy of the primary events. The upper threshold will probably depend on the energy of the incident photon. For events with energy be- low the Xenon $K$-shell the upper threshold will be set around $3.85 \mu \mathrm{s}$, narrowly around the peak of bl versus energy distribution. For events above the Xenon $K$-shell the upper thresholds has to be set to maximise the signal to background ratio which will depend on the in orbit HPGSPC background.

\subsection{Results on position reconstruction and energy correction}

Due to the hexagonal symmetry of the HPGSPC the lookup table need in principle be determined only in a $30^{\circ}$ degrees sector that is assumed as reference sector and then extended to the entire area of the instrument with an appropriate set of rotations and reflections. The physical symmetry of the HPGSPC was verified by sampling all the sectors using the response to the $22 \mathrm{keV}$ peak line of $\mathrm{Cd}^{109}$ in 20 different points per sector. Only the reference sector was carefully mapped in 120 points. Figures 10,11,12

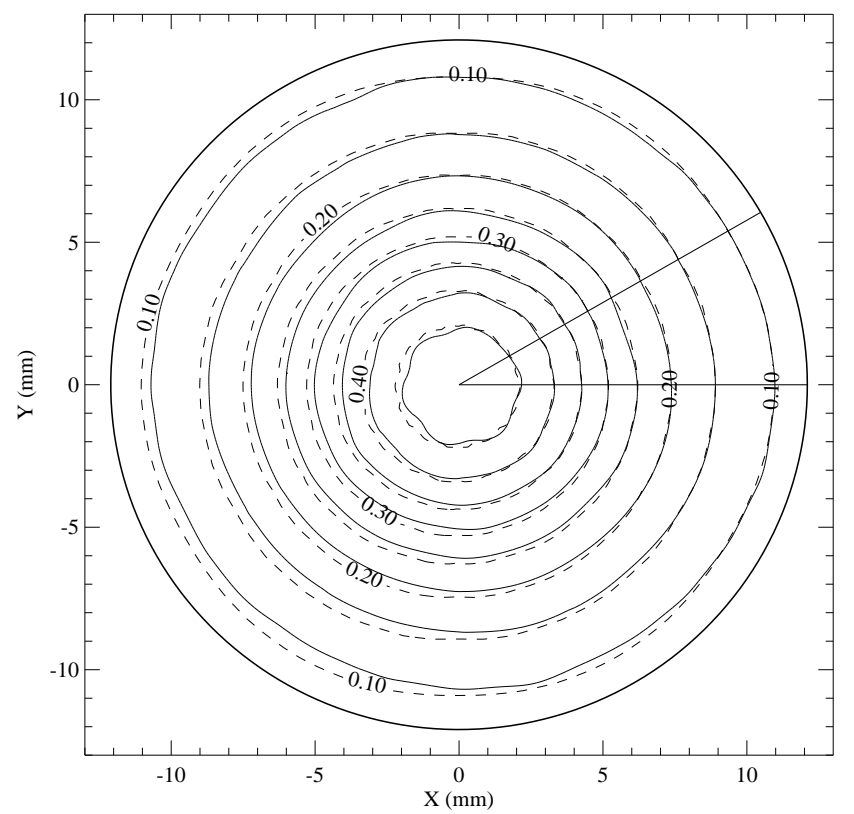

Fig. 10. Isocontours of the $C_{0}$ coefficient relative to the whole detector open area. Dashed lines are from experimental mapping in all sectors, while continuous lines are generated with a symmetrical mapping of the reference sector

show the isocontours relative to $C_{0}, C_{1}$ coefficients and to the $K_{\mathrm{c}}$ correction factor. The dashed lines plot is obtained from the experimental measurements, while a continuous line map is generated with a symmetrical mapping of the reference sector on the whole area of the detector. While for the $C_{1}$ coefficient the two mapping are in good agreement (differences are less than $2 \%$ ), showing that the gains 


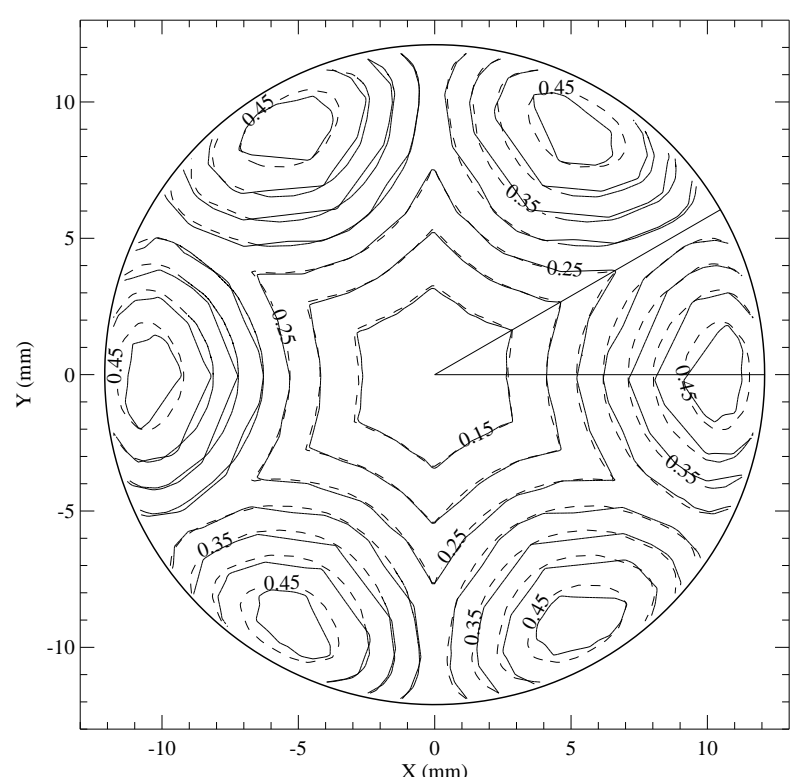

Fig. 11. Isocontours of the $C_{1}$ coefficient. The two mapping are in good agreement showing that lateral PMTs were correctly equalised

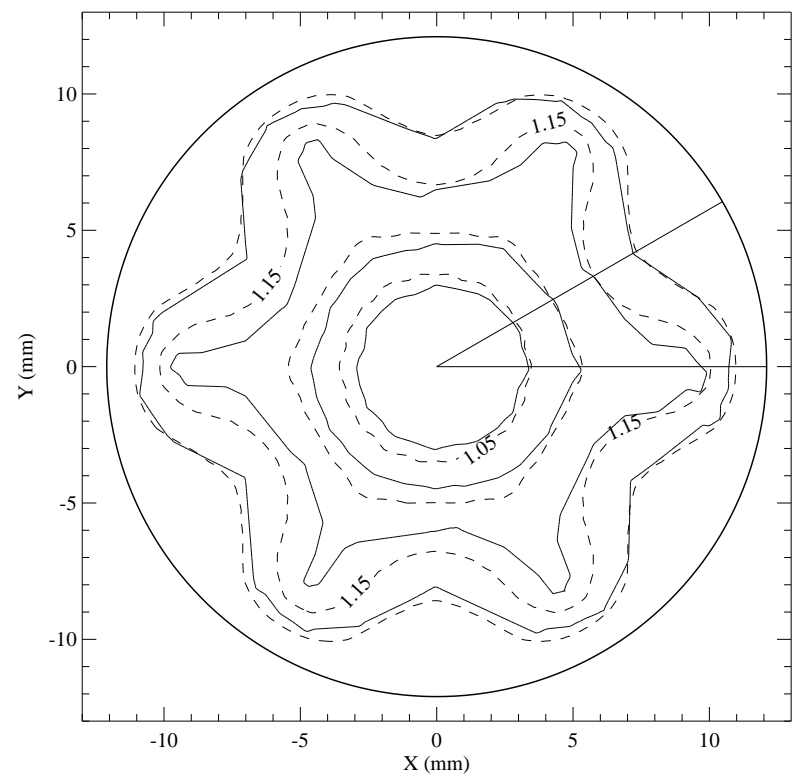

Fig. 12. The $K_{\mathrm{c}}$ correction factor isocontours: asymmetry is clearly visible of the lateral PMTs were correctly equalised, the asymmetry in the $K_{\mathrm{c}}$ contours is a consequence of the shift present in the $C_{0}$ contours. This is due to a misalignment between the geometrical centre of the detector and the optical centre of the central PMT. Simulation show that even a quite small misalignment (less than $1 \mathrm{~mm}$ and/or 1 degree) can have an impact (up to $10 \%$ for $\mathrm{r}$ greater than $10 \mathrm{~cm}$ ) in the Kc factor in some of the sectors. The imperfect reconstruction in these sectors affects the energy resolution and gives rise to a deviation from the gaussian statistical distribution of the reconstructed lines.

To minimise the effect of the asymmetry, we derived directly a $\left(C_{0}, C_{1}\right)$ distribution for a long measurement of a $\mathrm{Am}^{241} 59.5 \mathrm{keV}$ source illuminating the full area of the entrance window (see Fig. 13). Of course, the $\left(C_{0}, C_{1}\right)$ distribution does not depend on the energy of the calibration line used.

The $C_{0}$ constant map can then be obtained just by subdividing the $\left(C_{0}, C_{1}\right)$ coefficient distribution in rectangular areolas whose vertices belongs to $C_{0}$ isolines, (see Fig. 13).

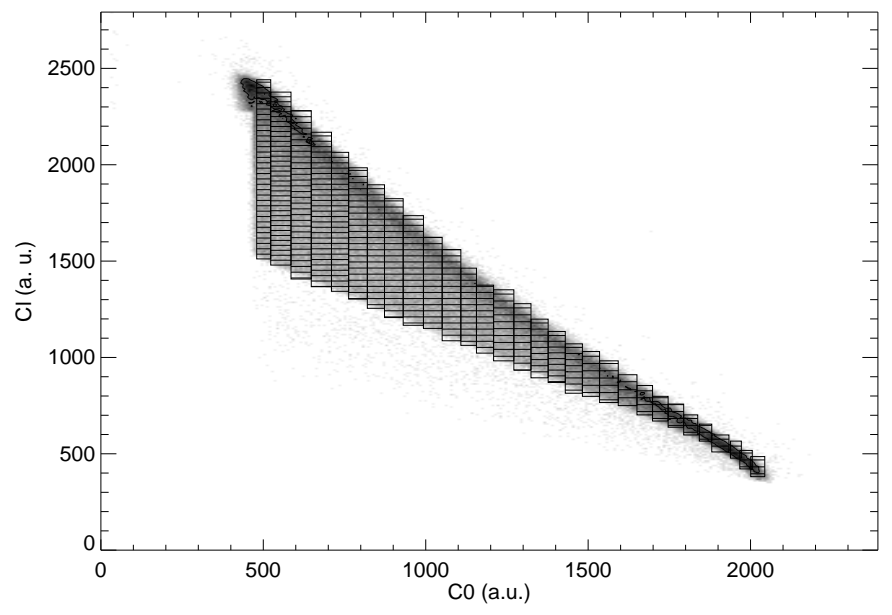

Fig. 13. The $\left(C_{0}, C_{1}\right)$ distribution for the $59.5 \mathrm{keV}$ line of $\mathrm{Am}^{241}$

For each areola $i$ the $K_{\mathrm{c}}$ factor can be defined as:

$K_{\mathrm{c}}(i)=\frac{\operatorname{Peak}(0)}{\operatorname{Peak}(\mathrm{i})}$

where $K_{\mathrm{c}}(i)$ is the correction factor of areola $i$, Peak $(0)$ is the peak of the $59.5 \mathrm{keV}$ line of $\mathrm{Am}^{241}$ in the energy spectrum collected in the central areola and $\operatorname{Peak}(i)$ is the peak of the $59.5 \mathrm{keV}$ line of $\mathrm{Am}^{241}$ in the energy spectrum collected in the $i$ areola. It must be stressed that the effects of asymmetry are averaged because photons collected in each $\left(C_{0}, C_{1}\right)$ areola are indeed physically detected in $(x, y)$ positions, which differ from sector to sector. 
The areolas are iteratively determined in such a way that a finer discretisation does not improve the energy resolution in the local $\left(C_{0}, C_{1}\right)$ region. As a consequence, the variation of the correction factor $\Delta K_{\mathrm{c}}$ has been determined to be less than $1 \%$ in the outer areas of the $\left(C_{0}\right.$, $C_{1}$ ) distribution while $\Delta K_{\mathrm{c}}$ is less than $0.5 \%$ in the inner region (where the local energy resolution is better due to the higher yield).

The correct working of the energy reconstruction algorithm is a key point for the optimum scientific operation of the HPGSPC. This is illustrated in Fig. 14 in which both the unreconstructed and reconstructed full area raw energy spectra of a $\mathrm{Am}^{241}$ radioactive source, are shown. The source was positioned 3 meters from the entrance beryllium window in order to illuminate the whole entrance area of the detector. Both single and correlated events are accumulated.

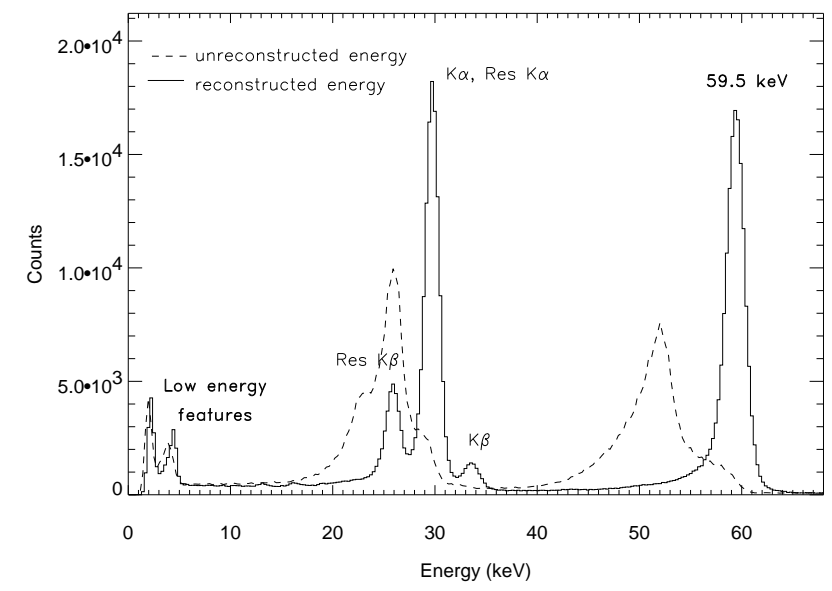

Fig. 14. Reconstructed and unreconstructed energy spectra of $\mathrm{Am}^{241}$ radioactive source

The unreconstructed spectrum is strongly distorted, shifted towards the lower channels and the lines are not well resolved. In the reconstructed raw spectrum the $\mathrm{Am}^{241} 59.5 \mathrm{keV}$ line is clearly detected and peak position is correctly reconstructed. The three, rather well resolved, lines at $25.7 \mathrm{keV}, 29.7 \mathrm{keV}$ and $33.8 \mathrm{keV}$ correspond respectively to the $K_{\beta}$ residual peak $\left(E_{\mathrm{x}}-K_{\beta}\right)$, the $K_{\alpha}$ fluorescence of the Xenon that overlap the $K_{\alpha}$ residual peak $\left(E_{\mathrm{x}}-K_{\alpha}\right)$ and the $K_{\beta}$ Xenon fluorescence. The two low energy features clearly visible in the spectrum below $10 \mathrm{keV}$ are due to $\mathrm{Am}^{241} 59.5 \mathrm{keV}$ photons absorbed directly in the Back Region which give rise to an electron cloud scintillating very close to the scintillation grid wires along anomalous paths. However as described in the previous paragraph this undesirable features can be almost totally rejected by burst length selection.

The HPGSPC resolving power in the entire operative range is shown in Fig. 15 where both unreconstructed and reconstructed energy spectra of a $\mathrm{Co}^{57}$ radioactive source are given.

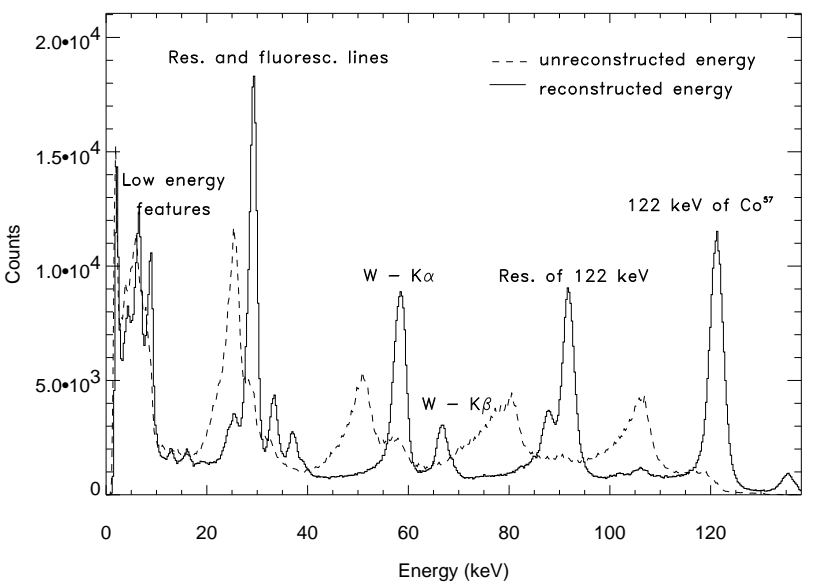

Fig. 15. Reconstructed and unreconstructed energy spectra of $\mathrm{Co}^{57}$ radioactive source. Low energy features are due to events absorbed in the Back Region. $K_{\alpha}$ and $K_{\beta}$ lines, emission from Tungsten is also visible

Low energy features are quite enhanced as expected because of the longer penetration depth of higher energy photons. In the reconstructed spectrum two lines at $13.5 \mathrm{keV}$ and $16.8 \mathrm{keV}$ are visible. They are due to Uranium contamination ( $L_{\alpha}$ and $L_{\beta}$ lines) in the beryllium window.

\subsection{Spectroscopic performance}

In Fig. 16 we show the full area corrected energy spectrum of the $22 \mathrm{keV}$ and $25 \mathrm{keV}$ lines of a $\mathrm{Cd}^{109}$ radioactive source in which background has been subtracted and burst length thresholds have been applied. A double gaussian fit is also shown. The agreement is not perfect because of residual distortion of the reconstructed spectrum in some of the sectors and due to discretisation in the $K_{\mathrm{c}}$ look-up table that can introduce a deviation of the counting statistics from the poisson distribution. The deviation from a gaussian nature is modeled in the response matrix of the instrument. In Fig. 17 we report the full area energy corrected spectrum of an $\mathrm{Am}^{241}$ radioactive source after background subtraction and burst length selection. The spectrum has been accumulated in Single Mode. Line at $59.5 \mathrm{keV}$ contains, then, only events that have been detected as "Single". The tail towards lower energies is due to double events that scintillate in spatially separated centres but overlap in burst time and then give rise to a non correctly detected light production centre. The line resolution is $3.3 \%+/-0.1 \%$ FWHM. The other two peaks at $25.7 \mathrm{keV}$ and $29.8 \mathrm{keV}$ correspond to the Escape $K_{\alpha}$ and Escape $K_{\beta}$ i.e. to events for which the fluorescence 


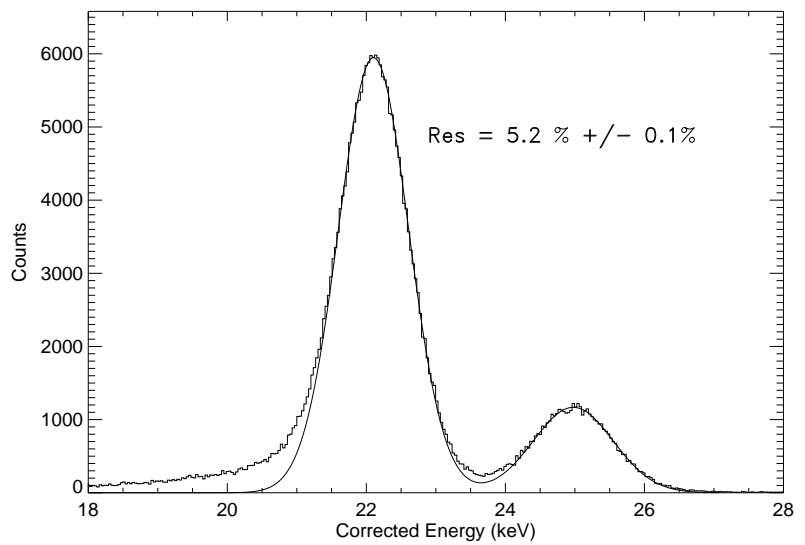

Fig. 16. Full area energy spectrum of 22 and $25 \mathrm{keV}$ lines of $\mathrm{Cd}^{109}$ radioactive source

photon exit from the detector. The low energy features relative to the events absorbed in the Back Region are much reduced in intensity. The Fluorescence Gated spectrum is shown in Fig. 18 (the background has been subtracted and burst length thresholds have been applied). Only events detected as "Double" are accepted if at least one of the events has an energy determined to lie within $+/-3 \sigma$ of either the $K_{\alpha}$ or $K_{\beta}$ fluorescence energy, and if this is the case, the exactly known energy of the $K_{\alpha}$ or $K_{\beta}$ line is summed to the measured energy of the residual. As can be seen in the figure, the energy resolution in FG mode is improved with respect to that obtained in SE mode by up to $2.4 \%$. The spectroscopic performances of

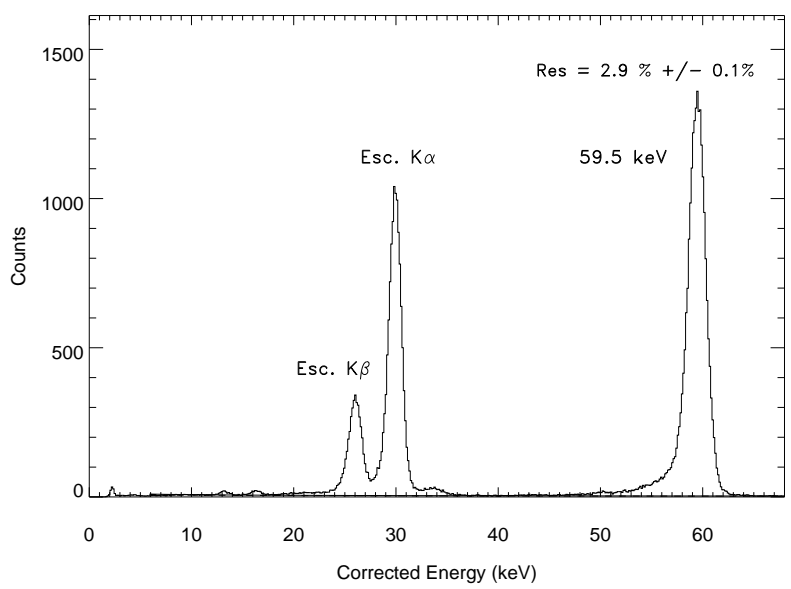

Fig. 17. Corrected energy spectrum of $59.5 \mathrm{keV}$ line of $\mathrm{Am}^{241}$ as seen by HPGSPC in Single Event Mode

the HPGSPC summarised in Fig. 19 where the energy resolution as a function of the energy is shown over the entire

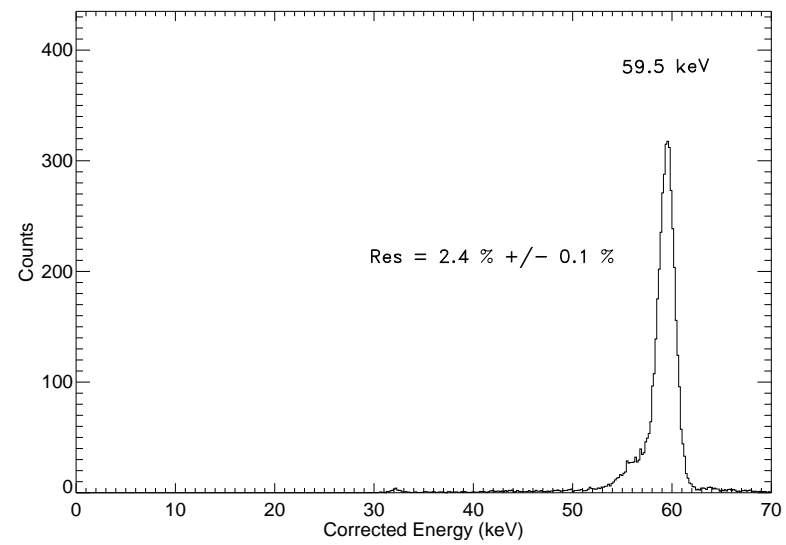

Fig. 18. Corrected energy spectrum of $\mathrm{Am}^{241}$ in Fluorescence Gated mode

energy range of the HPGSPC. Both SE and FG mode are shown. The figure also shows, for comparison, the energy resolution as a function of energy for narrowly collimated "on-axis" X-ray sources. The full area curve can be fitted with the following relation:

$$
\frac{\Delta E}{E}=3.30\left(\frac{E}{60 \mathrm{keV}}\right)^{-0.46}
$$

The deviation from the $E^{-0.5}$ law, observed with narrowly collimated illumination, results from the discretization (Giarrusso et al. 1989).

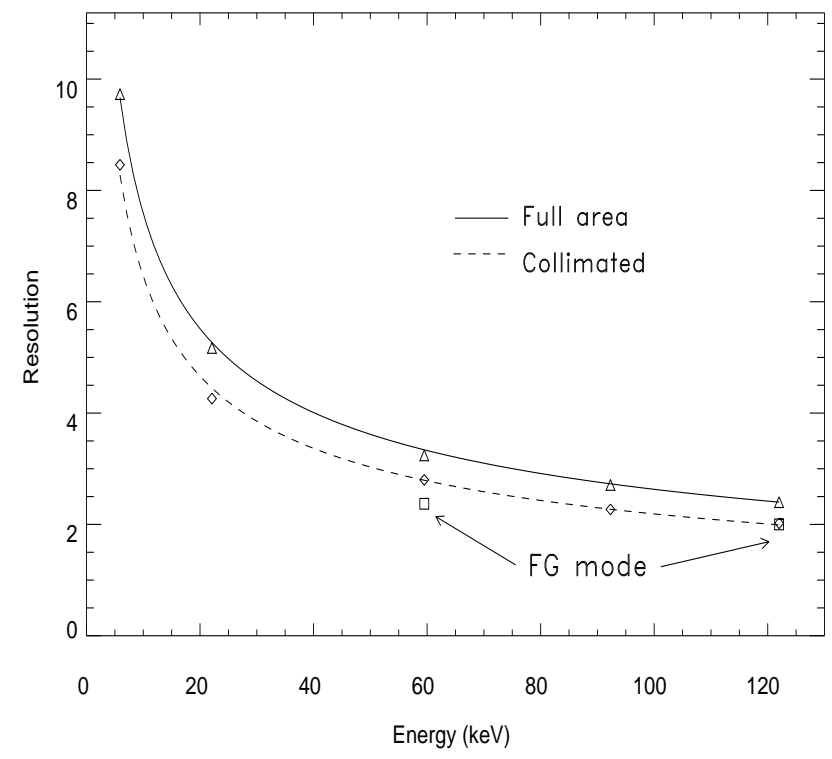

Fig. 19. Energy resolution versus energy: both full area and collimated case are given. Small squares show the energy resolution in FG mode 


\subsection{Energy linearity}

The detector's linearity has been verified for the response to both collimated and full area $\mathrm{X}$-ray illumination. Results are shown in Fig. 20 where all lines and residuals are reported. The channel to energy conversion is given:

$E=-0.16+0.0342 * \mathrm{PHA}$

for the SE mode and

$E=0.03+0.0342 * \mathrm{PHA}$

for the FG mode. The slight difference between the two mode is due to a small offset difference between the two electronic chains. Because of the limited number of energy lines we could not find any evidence for the gain discontinuity around the Xenon $K$-edge where a jump of $177 \mathrm{eV}$ has been recently measured (dos Santos G.M.F. et al. 1994). A $L$-jump discontinuity at $4.78 \mathrm{keV}$ of about $110 \mathrm{eV}$ has been also measured by many authors (dos Santos et al. 1993; Lamb et al. 1987; Boella et al. 1996). This effect is small for the HPGSPC, because, due to the window thickness, the detection efficiency at that energy is low. Both the $K$ and $L$ jump have been, however, modelled and introduced in the response characteristic of the detector.

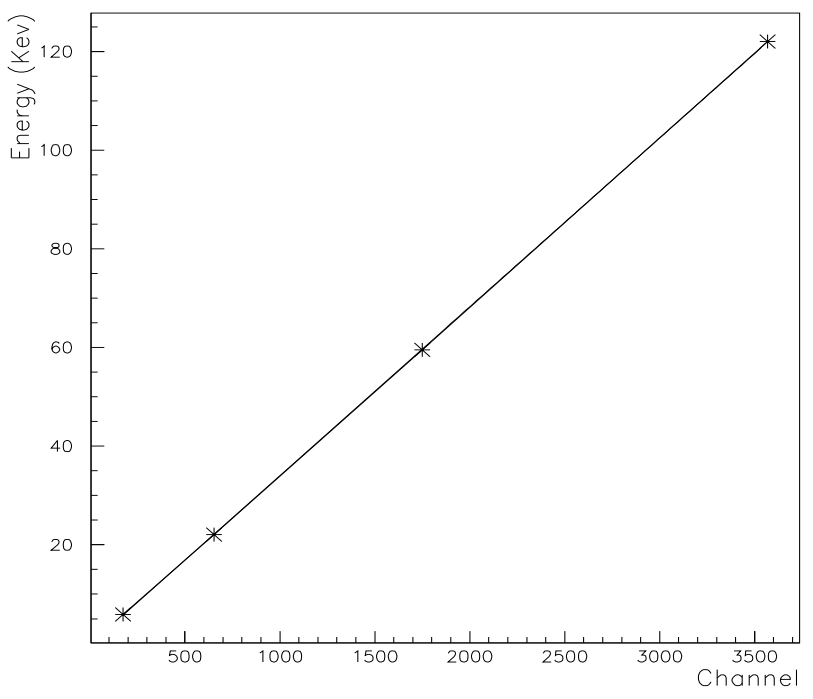

Fig. 20. Energy linearity

\subsection{Effective area and response matrix}

The effective area of the HPGSPC is mainly limited at low energies by the transmission of the beryllium entrance window, while at higher energies it is limited by the efficiency of absorption of X-rays in Xenon. in Fig. 21 we show the HPGSPC effective area as a function of energy. In the calculation have been taken into account the predicted transparency of collimator (around $75 \%$ ), the beryllium window transmission and the reduction factor due to the efficiency of electronics to recognise a true $\mathrm{X}$-ray.

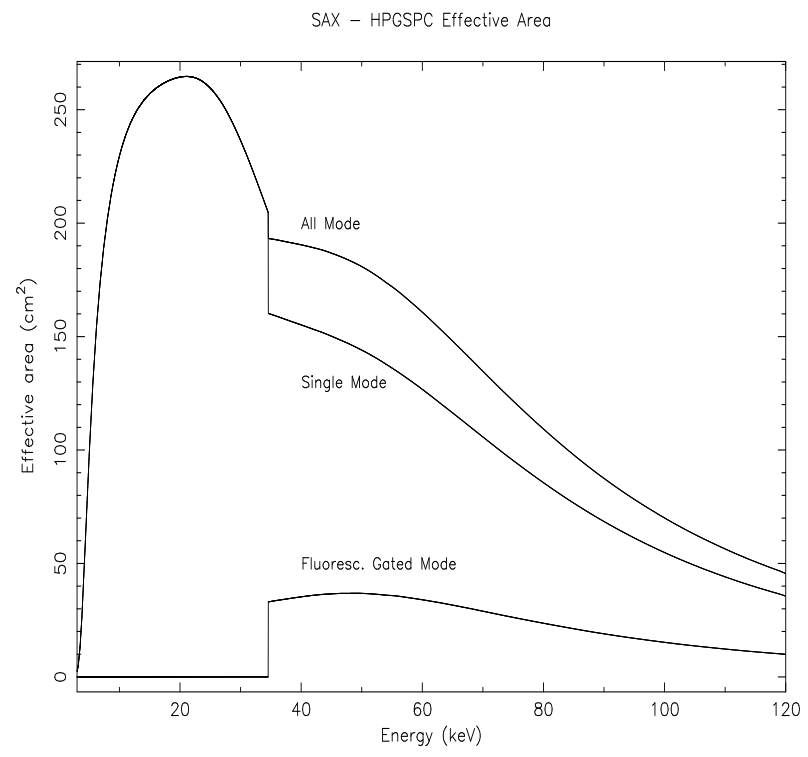

Fig. 21. HPGSPC effective area

In order to obtain the complete response matrix of the instrument, the redistribution matrix has been experimentally determined. The redistribution matrix depends, of course, on the energy resolution and on the distribution of the response to a monochromatic line, but it depend also (above $34.6 \mathrm{keV}$ ) on the escape fraction probabilities of $K_{\alpha}$ and $K_{\beta}$ fluorescence photons. Table 4 shows the experimentally determined escape fraction probabilities for the $K_{\alpha}$ and $K_{\beta}$ fluorescence photons for an incident energy equal to $59.5 \mathrm{keV}$ (Americium source) and to the 122 and $136 \mathrm{keV}$ of the $\mathrm{Co}^{57}$ lines. Escape probabilities have been normalized with respect to the total number of detected events in SE mode.

\subsection{Background rejection analysis}

Several components contribute to the background level of the HPGSPC. A major background source (Mason et al. 1983) is the diffuse isotropic atmospheric and Cosmic $\mathrm{X}$ and gamma ray component that manifests itself as an aperture flux that depends upon the Field of View of the collimator and as an indirect background induced through shield penetration. A second component is due to primary cosmic rays (mostly protons) and geomagnetically trapped 
Table 4. Escape probabilities for the Xenon $K_{\alpha}$ and $K_{\beta}$ fluorescence photons

\begin{tabular}{llr}
\hline Fluorescence Photon & energy & value \\
\hline$K_{\alpha}$ & full area $60 \mathrm{keV}$ & $27.4 \%$ \\
$K_{\beta}$ & full area $60 \mathrm{keV}$ & $9.1 \%$ \\
$K_{\alpha}$ & full area $122 \mathrm{keV}$ & $25.3 \%$ \\
$K_{\beta}$ & full area $122 \mathrm{keV}$ & $9.7 \%$ \\
\hline
\end{tabular}

particles that interact directly in the detector or, interacting with the spacecraft, giving rise to gamma rays which in turn penetrates the detector through the shielding and interacting with the detector walls or filling gas producing energetic Compton electrons. A third component is due to electrons and neutrons mainly produced as a result of the primary cosmic ray interaction with the atmosphere.

As the HPGSPC is a background dominated instrument for most sources, background reduction is extremely important. It is be achieved in three ways:

- Passive shielding. $1 \mathrm{~mm}$ of lead and $2 \mathrm{~mm}$ of tin cover all direction other than the field of view of the instrument.

- Burst length selection. As we already described only events that produce a VUV light burst whose duration is within the upper and lower thresholds (experimentally determined for $\mathrm{X}$-rays) are recognised as "genuine" events.

- Radius selection. Events detected outside a selected radius (maximum value is $12 \mathrm{~cm}$ ) are rejected as background events coming from the detector wall.

Precise simulation of the in-orbit background is practically impossible on the ground. However, to provide an insight into the background rejection capabilities of the HPGSPC two "on ground available" background sources have been used: the environmental background at Laben Clean Room (that is the sea level cosmic ray flux) and a $\mathrm{Co}^{60}$ gamma ray source $(1.17 / 1.33 \mathrm{MeV}$ gamma rays) that can mimic the in-orbit background component of energetic Compton electrons. In Fig. 22 we show the energy reconstructed spectrum of the background (both environmental and $\mathrm{Co}^{60}$ induced background) as seen by the HPGSPC.

In order to evaluate and optimise the background rejection efficiency of a X-ray detector, such as the HPGSPC the statistic $S$ defined as:

$S=\frac{A_{\mathrm{x}}\left(E_{\mathrm{x}}\right)}{\sqrt{1-R_{\mathrm{b}}\left(E_{\mathrm{x}}\right)}}$

must be maximised. In the previous equation $A_{\mathrm{x}}\left(E_{\mathrm{x}}\right)$ is the $\mathrm{X}$-ray acceptance efficiency i.e. the fraction of $\mathrm{X}$-rays, with energy $E_{\mathrm{x}}$, accepted by burst length selection with respect to the total number of $\mathrm{X}$-rays events recorded

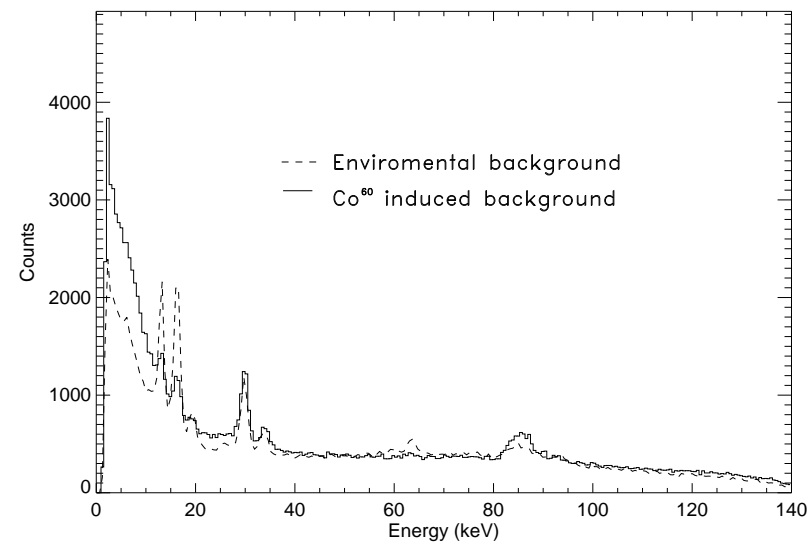

Fig. 22. Environmental and $\mathrm{Co}^{60}$ induced background as seen by the HPGSPC

by the HPGSPC and $R_{\mathrm{b}}\left(E_{\mathrm{x}}\right)$ is the fraction of background events rejected because of burst length selection with respect to the total background. The variation of burst length efficiency $R_{\mathrm{b}}$ with $A_{\mathrm{x}}$ is shown in Fig. 23 along with the Statistic S, for the $22 \mathrm{keV}$ line of $\mathrm{Cd}^{109}$ source. The two curves correspond to the environmental background at Laben and to a $\mathrm{Co}^{60}$ induced background. As $\mathrm{S}$ should be maximum, the optimum rejection efficiency $R_{\mathrm{b}}$ occurs at $55 \%$ with an acceptance $A_{\mathrm{x}}$ of $90 \%$.

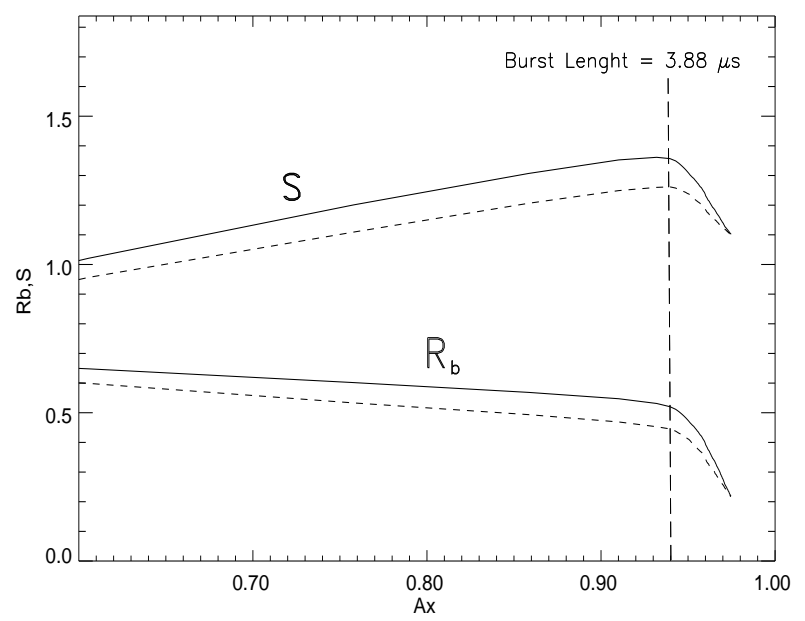

Fig. 23. Background rejected and Statistic S versus energy for $\mathrm{Cd}^{109}$ source: dashed lines correspond to environmental background; continuous line is for $\mathrm{Co}^{60}$ induced background. The vertical line corresponds to a choice of burst length window narrowly selected around the burst length of genuine $\mathrm{X}$-ray events 
Above $34.6 \mathrm{keV}$ the situation is different. In Fig. 24 the variation of $R_{\mathrm{b}}$ is shown for the $59.5 \mathrm{keV}$ line of $\mathrm{Am}^{241}$. A Maximum is obtained when the upper burst length thresholds is fully open. This is due to the long tail that is present in the Americium burst length spectrum. The vertical line in Figs. 23 and 24 corresponds to a choice of burst length window narrowly selected around the burst length of genuine $\mathrm{X}$-ray events. Finally in Fig. 25 we show the burst

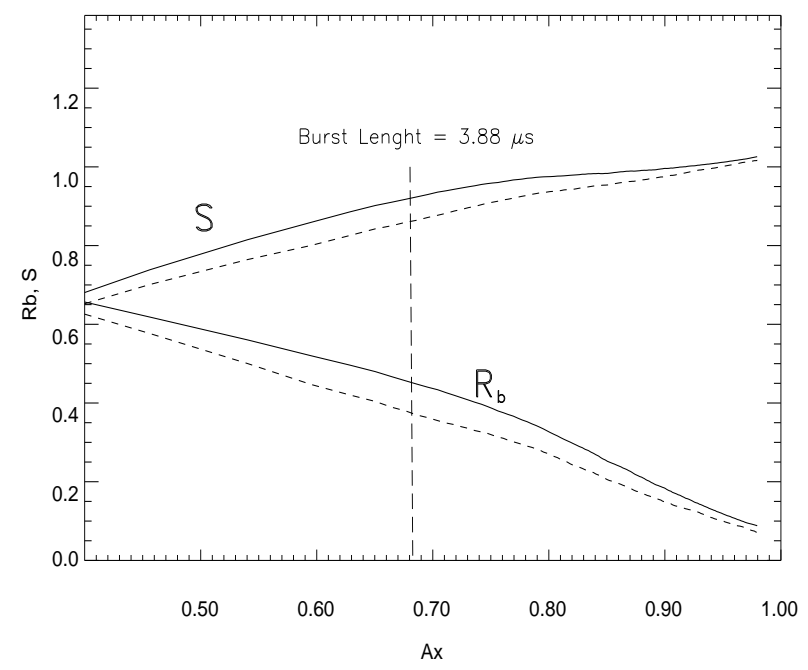

Fig. 24. Background rejected and statistic $S$ versus energy for an $\mathrm{Am}^{241}$ source

length rejection efficiency as a function of X-ray photon energy for an X-ray acceptance efficiency of $80 \%$. Only events with energies within of the $\mathrm{X}$-ray lines are considered.

The background rejection efficiency $R_{\mathrm{k}}$ in Fluorescence Gated mode has been measured using both a $\mathrm{Co}^{60}$ gamma ray source and the environmental background (Sims et al. 1983). $R_{\mathrm{k}}$ has been measured in a narrow band around the reference energy and the results are shown in Table 5 .

Several components contribute to the residual FG background. First, fluorescent photons can be produced by the interaction of charged particles (mostly protons and Compton electrons) with the Xenon $K$ shell electrons (Manzo et al. 1980; Middleman et al. 1967). Secondly, Compton scattered gamma rays have a $4 \%$ probability of producing fluorescence photons by interacting with the Xenon $K$-shell electrons. However, only a minor difference has been found between the $\mathrm{Co}^{60}$ and cosmic ray induced backgrounds. This suggests, in good agreement with Sims et al. (1983) and Ramsey et al. (1990), that, for the HPGSPC, the majority of the FG background is produced by high energy photons which scatter many times in the detector shielding and body and are ultimately absorbed in the Xenon via photoelectric interaction, show-

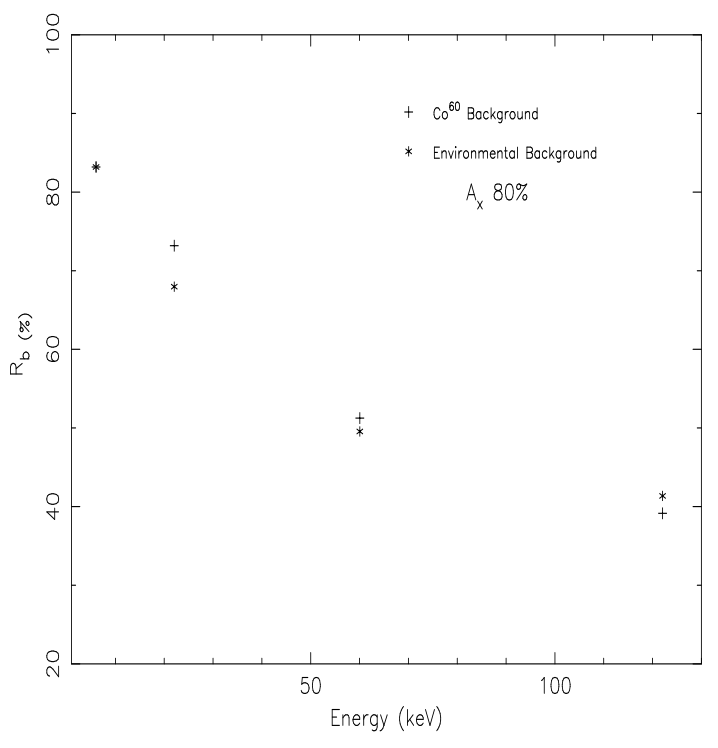

Fig. 25. Burst length versus energy for an $\mathrm{X}$-ray acceptance efficiency of $80 \%$

ing the same indistinguishable "fingerprint" as genuine $\mathrm{X}$-rays. An estimate of the influence of the above described components is currently being determined. Table 5 summarises, the background rejection efficiency at various incident photon energies $E_{\mathrm{x}}$ using both burst length discrimination and the FG technique.

Table 5. Rejection efficiency

\begin{tabular}{llll}
\hline Background & Ref energy & $R_{\mathrm{k}}$ & $R_{\mathrm{kb}}$ \\
\hline Co $^{60}$ & $40 \mathrm{keV}$ & $91 \%$ & $98 \%$ \\
$\mathrm{Co}^{60}$ & $60 \mathrm{keV}$ & $92 \%$ & $94 \%$ \\
Co $^{60}$ & $90 \mathrm{keV}$ & $93 \%$ & $95 \%$ \\
Env. bck. & $40 \mathrm{keV}$ & $92.9 \%$ & $98 \%$ \\
Env. bck. & $60 \mathrm{keV}$ & $85.4 \%$ & $93 \%$ \\
Env. bck. & $90 \mathrm{keV}$ & $90.9 \%$ & $92.7 \%$ \\
\hline
\end{tabular}

\section{Post launch addendum}

On April 30th 1996 BeppoSAX was successfully launched from Cape Canaveral. Following launch the satellite was commissioned for two months. On June 221996 the HPGSPC made its first observation of a celestial 
$\mathrm{X}$-ray source the black-hole candidate Cyg $\mathrm{X}-1$. At the time of writing the HPGSPC is completing the Science Verification Phase observing a number of well known $\mathrm{X}$-ray source.

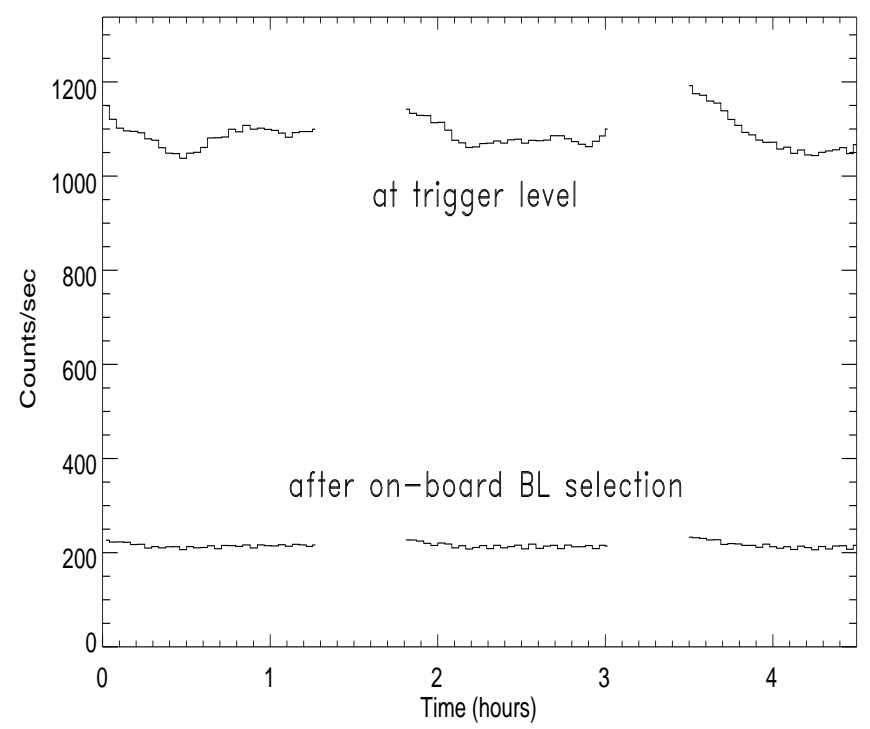

Fig. 26. Background count rate observed by the HPGSPC during in flight operation. Three consecutive orbits are shown. The upper curve refers to the count rate measured at the trigger while the bottom curve shows the residual background after on-board burst length selection

Characterisation of background is one of the main goal of the Science Verification Phase. Very preliminary results show that while the count-rate at the Trigger level is about a $10^{3}$ counts/sec after on-board burst length selection the background is reduced to $210^{2}$ counts/sec. Moreover, as can be seen in Fig. 26 the background is clearly modulated with the orbit. If the FG technique is applied the background count rate is reduced to $3.510^{-1}$ counts/sec in the $40-60 \mathrm{keV}$ band, that means a flux of $210^{-4}$ that confirms what expected on the basis of the on-ground calibration. Unfortunately the background spectrum, reported in Fig. 27, is highly structured. This is essentially due to a imperfect subtraction of the photons coming from the calibration sources. We are currently working to optimally configure the instrument in order to reduce this effect. A complete in-flight characterisation of the instrument and its performances will be subject of a future paper.

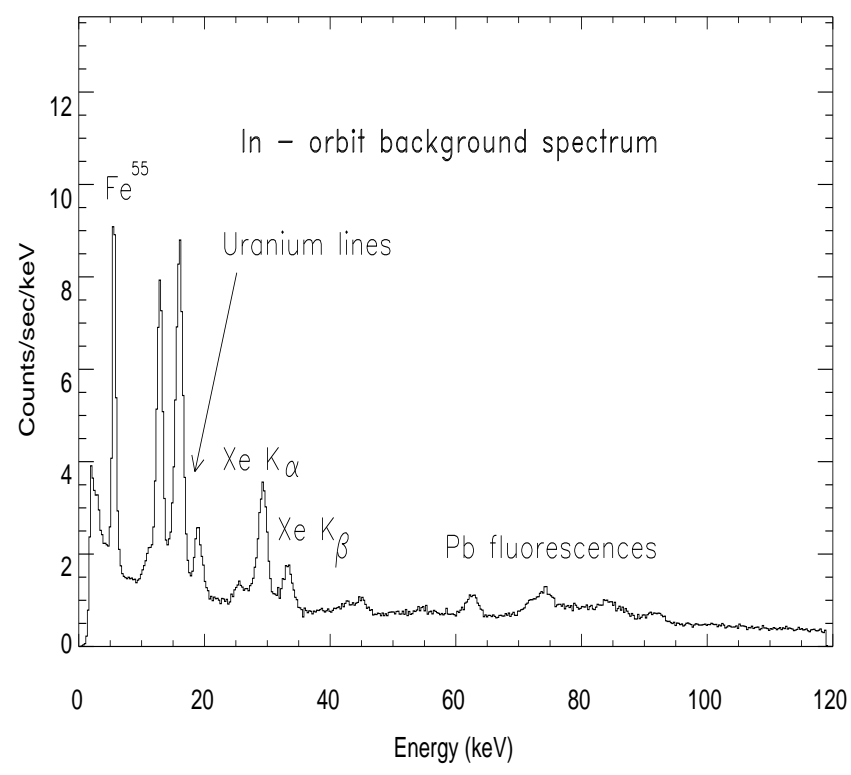

Fig. 27. Background spectrum as observed by the HPGSPC during the Commissioning Phase. The $\mathrm{Fe}^{55}$ line is due to events from calibration sources not well subtracted. $\mathrm{Pb}$ fluorescences are due to the $88 \mathrm{keV}$ component of the $\mathrm{Cd}^{109}$ calibration source

\section{List of acronyms}

AGC Automatic Gain Control

ASI Agenzia Spaziale Italiana (Italian Space

DU Agency)

EU Electronic Unit

FEE Front End Electronics

FG Fluorescence Gated

GSPC Gas Scintillation Proportional Counter

HPGSPC High Pressure Gas Scintillation Proportional Counter

ITE Instrument Test Equipment

LECS Low Energy Concentrator System

MECS Medium Energy Concentrator System

NFI Narrow Field Instrument

OBDH On Bord Data Handling

PDS Phoswich Detection System

PMT Photomultiplier

SAX Satellite Astronomia X

VUV Vacuum Ultra Violet

WFC Wide Field Cameras.

Acknowledgements. This research has been fully supported by the Italian Space Agency in the framework of the SAX program. We wish to thank Prof. L. Scarsi, Chairman of the SAX Scientific Steering Committee and Dr. Chris Butler, 
SAX payload manager, for their continuous support and encouragement.

\section{References}

Anderson D.F., Hamilton T.T., Ku W. H., et al., 1979, Nucl. Inst. Meth. 163, 125

Boella G., Chiappetti L., Conti G., et al., 1995, Proc. SPIE 2517,223

Dangendorf V., Schmidt-Böcking H., Breskin A., et al., 1989, Proc. SPIE 1159, 192

Dos Santos G.M.F., Bento A.C., Conde C.A., et al., 1994, Nucl. Inst. Meth. 350, 316

Frontera F., Butler C., Costa E., et al., 1995, Proc. SPIE 2517, 249

Gedanken A. Jorter J., Raz B,. et al., 1972, J. Chem. Phys. 57,3456

Giarrusso S., La Rosa G., Manzo G., et al., 1989, Proc. SPIE 1159,514

Giarrusso S., Santangelo A., Fazio G., et al., 1995a, Proc. SPIE 2517,234

Giarrusso S., Manzo G., Santangelo A., et al., 1995b, Nucl. Inst. Meth. A 354, 567

Jager R., Heise J., Mels W.A., et al., 1995, Proc. SPIE 2517, 261

Johnson N., et al., 1994, Proc. of the 2nd Compt. Obs. Symp.
In: Fichtel C.E. et al. (eds.) New York, p. 515

Leimann E.A., Van Dordrecht A., 1977, Nucl. Inst. Meth. A 146,399

Manzo G., Davelaar J., Peacock A., et al., 1980, Nucl. Inst. Meth. A 177, 595

Makishima K., Mihara T., Nagase F., et al., 1991, Proc. of 28th Yamada Conference on Frontiers of X-ray Astronomy. In: Tanaka Y. and Koyama K. (eds.)

Martin D.D.E., Bavdaz M., Peacock A., et al., 1995, Proc. SPIE 2517, 209

Mason I.M., Culhane J.L., 1983, IEEE Trans. Nucl. Sci. 30, 485

Nguyen Ngoc H., Jeanjean J., Itoh H., et al., 1980, Nucl. Inst. Meth. 172, 603

Piro L., Scarsi L., Butler C., 1995, Proc. SPIE 2517, 169

Ramsey B.D., Bower C.R., Dietz K.L., et al., 1990, Proc. SPIE 1344,82

Sims M.R., Manzo G., Peacock A., Taylor B.G., 1983, Nucl. Inst. Meth. 211, 499

Scarsi L., 1993, A\&A 97, 371

Taylor B.G., Andresen R.D., Davelaar J., et al., 1981, IEEE Trans. Nucl. Sci. 28, 861

Trümper J., Pietsch W., Reppin C., et al., 1978, ApJ 219, L105

Wheaton Wm.A., Doty J.P., Primini F.A., et al., 1979, Nat 282,240 